\title{
INNOVATIVE ALGORITHM TO EVALUATE THE CAPABILITIES OF VISUAL, NEAR INFRARED AND INFRARED TECHNOLOGIES FOR THE DETECTION OF VEINS FOR INTRAVENOUS CANNULATION
}

\author{
Maryam Asrar, ${ }^{1}$ Amin Al-Habaibeh, ${ }^{2, *}$ Mohammed Houda $^{3}$ \\ ${ }^{1,2}$ Product Design, Nottingham Trent University, Nottingham, NG1 4BU, UK \\ ${ }^{3}$ Consultant Obstetrician and Gynaecologist, Airedale General Hospital, Steeton, West Yorkshire, UK \\ *Corresponding author: Amin.Al-Habaibeh@ntu.ac.uk
}

\begin{abstract}
Intravenous cannulation is the process of inserting a cannula into a vein to administrate medication, fluids or to take blood samples. The process of identification and locating of veins plays an important role during the intravenous cannulation procedure to reduce health care costs and suffering of patients. This paper compares between the three technologies to assess their suitability and capability for the detection of veins to support the cannulation process. Three types of cameras are used in this study, a visual, an infrared and a near infrared. The collected images, 103 I total, from the three technologies have been analysed using a wide range of image processing techniques and compared with identification templates to evaluate the performance of each technology. The results show that the near infrared technology supported by suitable LED illumination is the most effective for the visualisation of veins. However, infrared thermography is found to be successful when followed by a cold stimulation.
\end{abstract}

OCIS codes: (100.2000) Digital image processing; (110.0110) Imaging systems; (120.3890) Medical optics instrumentation; (130.3060) Infrared; (040.2480) FLIR, forward looking infrared; (110.3080) Infrared imaging; (100.2980) Image enhancement.

\section{INTRODUCTION}

Peripheral intravenous cannulation is the procedure of inserting a cannula into the peripheral veins, in most cases the veins of the hand or forearm. It is used for many medical procedures such as maintaining hydration, administering blood or blood components and administering drugs such as antibiotics [1]. Numerous studies have identified the difficulties faced by clinical staff to perform intravenous cannulation [2]. For example, in the USA it is estimated that more than 400 million intravenous (I.V.) catheters are used daily to deliver medicine in the USA with success rate of about $72.5 \%$ in the first attempt [3].

The use of several attempts for intravenous cannulation increases the suffering of patients, and could cause damage to veins and neighbouring tissues. Therefore, it is vital to setup the route of the peripheral vein effectively on the first attempt. Astonishingly, there is presently limited literature regarding the visibility of veins in patients or the patient characteristics associated with difficult IV access. Earlier studies reported a range of aspects that influence vein visibility needed for vein cannulation [4 - 7]. Patients who have difficult venous access are a major challenge for modern medical care. A patient's level of hydration influence the ability to identify their veins. If the patient is obese, normal cues are typically absent making venous access enormously complex. Paediatric patients bring their own challenges with smaller vessels. The current study is an innovative approach for comparing the three technologies visual, near infrared and infrared for identification of the veins for intravenous cannulation using scenarios of different characteristics.

The behaviour of human skin changes between the visible and near infrared (NIR) ranges. Within the NIR range, scattering becomes of key significance in deeper skin layers while skin pigments (melanin) become less significant [8]. Hence, the penetration depth of the optical radiation in the NIR spectrum can become significantly larger than of the visible light. Therefore, skin images of people recorded in NIR spectrum give accurate details of the blood vessels at shallow depth below the surface of the skin in comparison to visual cameras. NIR 
radiation can easily penetrate into the depth of blood vessels and the size and location of the veins can be easily visualised in this case. According to [8], high power NIR LEDs with wave length of about 850 $\mathrm{nm}$ produce the best result in this case.

Infrared thermography can also be used to detect veins since blood flow causes a major effect on skin temperature distribution by mainly thermal convection and conduction. According to [9], the transfer of heat mechanisms through blood flow is influenced by gravity opposition to blood flow. However, the use of infrared thermography would require a cold or warm compress to provide the temperature variation to ease visualisation.

For finding three-dimensional information for identification of blood vessels location and depth, near-infrared (NIR) optical imaging devices have been reported to be effective $[10,11]$. This can be done without the need of any cold or warm compress (cold or warm stimulation). Traditionally, infrared thermometry is used to measure skin temperature [13], however, the application of vein visualisation is still limited in industry and this paper will assess infrared thermography in comparison to near infrared and visual images. It has been argued that the best NIR wavelengths to optimise vein contrast are determined experimentally [14]. On the other hand, infrared thermography can measure skin temperature [15] and hence light illumination will not be needed.

\section{A. Problem statement}

- The major problem faced by the clinical staff is the difficulty in accessing veins for intravenous drug delivery. With improper detection of veins, several problems such as bruises, rashes, blood clot etc. could occur.

- Subcutaneous fat or dark skin colour reduces the visualization of blood vessels underneath the skin.

- Gaining intravenous access in children can be difficult.

To address the above mentioned problems due to improper vein detection especially in the patient characteristics associated with difficult IV access there is a strong need to develop a vein locating device with suitable technology and simple image processing techniques.

The present research work is an attempt to design an algorithm to evaluate the capabilities of three different technologies for the detection of veins for IV access. This paper is aiming to compare between visual, infrared and near infrared images for the detection of veins and to establish an easy, compact, safe and reliable visualization device with minimal interference in the usual routine of vessel puncturing.

\section{B. Vein locating systems}

Many vein locators are currently available in research and industry to improve vascular access, see Table 1 . The current vein locating systems available for this purpose in the market have some limitations, drawbacks and are somehow costly. There are various challenges to be found throughout the design and implementation of a device such as the lighting system, the image processing algorithms, the physical design and the cost.

For medical imaging, infrared cameras are becoming less expensive providing high flexibility for different types of applications. Image processing techniques can be used to provide a wide range of effective medical and other industrial applications [16]. Near infrared cameras can be developed cheaply with the modification of visual cameras through filter modifications. However, many modern cameras are available now with simple optical filters for the near infrared spectrum [8].
Although, a few devices based on the infrared technique have been implemented, there still exists a strong need to develop such medical devices. Table 1 presents a summary of the most common vein finding systems available in industry.

To visualise the veins accurately there are limitations in relation to capabilities of different vein locating systems presently available in the market. The near infrared vein locators or viewers normally would produce good results in ideal conditions such as low fat content and clear skin. But, due to complex relationship between various physiological parameters, the performance can differ. This becomes also difficult when estimation of veins' depth or diameter is needed [32]. For medical applications, the standardisation of protocols is essential, and this must also be applied to the image processing and the selection of repeatable regions of interest [33].

Other technologies such as radiography can be used to obtain clear images of vessels with diameters of $200 \mu \mathrm{m}$ or less cannot be obtained by conventional radiography [34], however, this technology is beyond the focus of this paper.

The main focus of this study is on the detection of veins and the enhancement of visualisation using different camera technologies and image processing techniques.

Table 1. Vein Locators with their Working Methods, Approximate Comparative Prices ${ }^{1}$ and Schematic Diagrams

\begin{tabular}{|c|c|c|c|c|}
\hline $\begin{array}{l}\text { Vein Locator } \\
\text { Systems }\end{array}$ & Company & Working Method & $\begin{array}{l}\text { Price Indication } \\
\text { (App rox.) }\end{array}$ & Schematic Presentation \\
\hline $\begin{array}{c}\text { Accul ein } \\
\text { AV } 300 \text { V ein } \\
\text { Viewring System }\end{array}$ & $\begin{array}{c}\text { AccuV ein } \\
\text { (1) }\end{array}$ & $\begin{array}{c}\text { N ear infrared light } \\
\text { is used to locate } \\
\text { peripheral veins } \\
\text { beneath the surface } \\
\text { of the skin. [17] }\end{array}$ & $\begin{array}{l}\text { US } \$ 2,000 \\
{[18]}\end{array}$ & \\
\hline $\begin{array}{l}\text { Vein locator } \\
\text { BS2000+ }\end{array}$ & $\begin{array}{c}\text { Wrxi } \\
\text { Belson } \\
\text { Medical } \\
\text { System } \\
\text { Co.,LTD } \\
\end{array}$ & \begin{tabular}{|c|} 
Vein locator \\
BS2000+ uses near \\
infr ared light and \\
LED as light source. \\
{$[19]$}
\end{tabular} & $\begin{array}{c}\text { US } \$ 5,000 \\
{[20]}\end{array}$ & \\
\hline Veinlite(1) & $\begin{array}{l}\text { Wartior } \\
\text { Edge, LLC }\end{array}$ & $\begin{array}{c}\text { It works by } \\
\text { illuminating the de- } \\
\text { oxygenated blood in } \\
\text { veins. [21] }\end{array}$ & $\begin{array}{r}\$ 629 \\
{[22]}\end{array}$ & \\
\hline $\begin{array}{c}\text { Economical } \\
\text { Dualhead Vein } \\
\text { 1ocator BM1000 }\end{array}$ & \begin{tabular}{|c|} 
Whrsi \\
Belson \\
Medical \\
System \\
Co.,LTD \\
\end{tabular} & $\begin{array}{c}\text { It uses near infrared } \\
\text { light source. [23] }\end{array}$ & $\begin{array}{c}\$ 1,050 \\
{[24]}\end{array}$ & \\
\hline VascuL uminator & DKMP bv & $\begin{array}{c}\text { It works with the } \\
\text { help of near infr ared } \\
\text { light source. [25] }\end{array}$ & $\begin{array}{l}\text { US } \$ 10,000 \\
{[26]}\end{array}$ & \\
\hline $\begin{array}{l}\text { Luminetx } \\
\text { VeinViewer }\end{array}$ & Luminetx & \begin{tabular}{|c|} 
A near-infrared \\
LED source \\
differentiates red \\
blood cells of \\
subcutaneous veins \\
from surrounding \\
tissues and arteries. \\
{$[27]$} \\
\end{tabular} & $\begin{array}{c}\$ 25,000 \\
{[28]}\end{array}$ & \\
\hline $\begin{array}{c}\text { Veinsite hands- } \\
\text { free system }\end{array}$ & \begin{tabular}{|c|} 
VueTek \\
Scientific
\end{tabular} & \begin{tabular}{|c|} 
It uses near infrared \\
light to image \\
superficial veins to \\
a depth of $7 \mathrm{~mm}$. \\
{$[29]$}
\end{tabular} & $\begin{array}{c}\$ 4,595 \\
{[30]}\end{array}$ & \\
\hline \begin{tabular}{c|} 
Nottinghamm \\
Trent University
\end{tabular} & $\mathrm{N} / \mathrm{A}$ & \begin{tabular}{|c|} 
It can use near \\
infrared and/or \\
infrared with mobile \\
phone technology. \\
{$[31]$}
\end{tabular} & Not in the market yet. & \\
\hline
\end{tabular}

\section{METHODOLOGY}

Schematic diagram of the methodology is shown in Figure 1. Case studies have been selected from a pool of volunteers to present extreme difference in characteristics associated with the veins clarity,

1 The price figures are approximate comparative values and should not be considered as an exact price or an accurate quotation due to significant variability in the options and pricing. 
varying from cases with very visible veins and other cases that are characterised by challenging veins with less visibility. The visual $(\mathrm{N}=$ $38)$, near infrared $(\mathrm{N}=40)$ and infrared $(\mathrm{N}=25)$ images are captured of volunteers with different skin tones, ethnicities, weights, gender and age groups (19 - 55). The captured images are processed by different image processing techniques to enhance the visualisation of the veins. Regions of interest are selected in all three types of captured images (raw data). This research paper presents a novel approach to calculate the error based on a combination between image processing and an identification template (vein map) for comparison.

On one hand, templates $(\mathrm{N}=103)$ are generated manually by most probable visible veins for all three types of cropped images (expert interpretation). After that, templates are converted into grayscale and thresholding is applied for comparison with processed images. On the other hand, a series of image processing techniques are applied on three types of cropped images $(\mathrm{N}=103)$ to enhance the visualisation of veins. To assess the efficiency of the three different technologies differences are found between templates and the processed images. Finally, errors are calculated (\% pixels) for all images of the three different types. Also, mean error (\% pixels) and standard deviation of errors (\% pixels) for each technology are found.

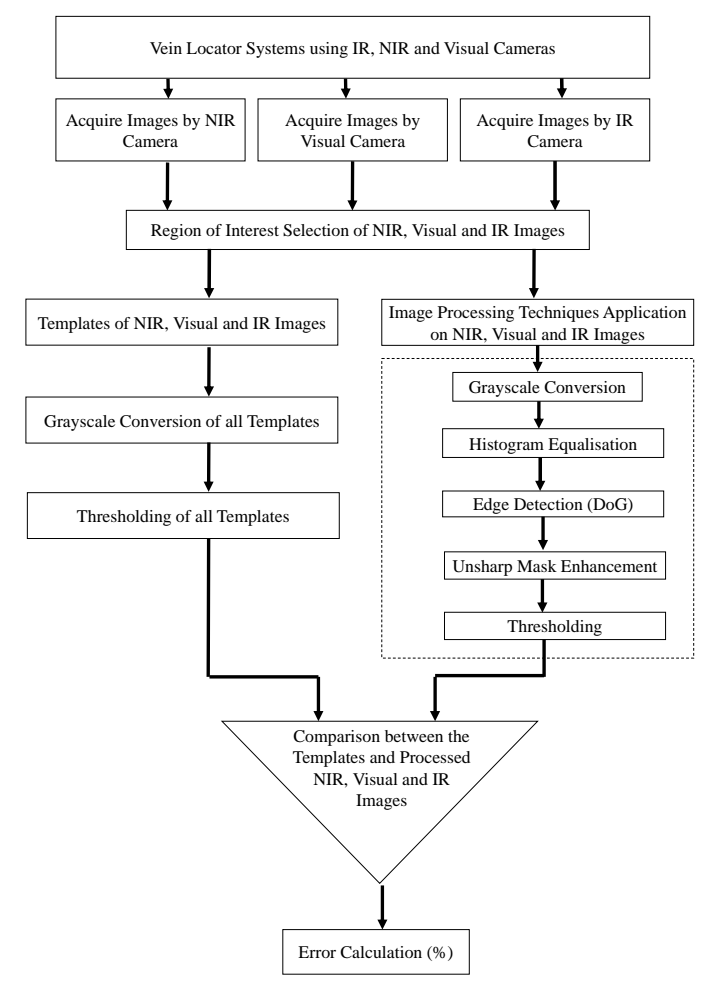

Fig.1. The implemented methodology of this research work.

\section{A. Image processing techniques}

Grayscale is used in this research work for transforming images from colour to grayscale. Average method is used to calculate the grey values. It calculates the mean of the red, green and blue colours values:

$$
(R+G+B) / 3
$$

Where $R, G$ and $B$ are the red, green and blue values of each pixel. Difference of Gaussians is also used. It is a feature enhancement algorithm that finds difference of lower type of actual blurred image from its higher type [36]. Mathematically, given an m-channels, ndimensional image

$$
I:\left\{X \subseteq R^{n}\right\} \rightarrow\left\{Y \subseteq R^{m}\right\}
$$

The difference of Gaussians (DoG) of the image $I$ is the function

$$
\Gamma_{\sigma 1, \sigma 2}:\left\{\mathrm{X} \subseteq R^{n}\right\} \rightarrow\{Z \subseteq R\}
$$

Histogram equalization is implemented in this research work. The contrast of many images is raised by histogram equalisation mainly when the usable data of the image is represented by close contrast values.

Consider a discrete grayscale image $\{x\}$ and let $n_{i}$ be the number of occurrences of gray level $i$. The probability of an occurrence of a pixel of level $i$ in the image is:

$$
p_{x}(i)=p(x=i)=\frac{n_{i}}{n}, 0 \leq i<L
$$

Where $L$ being the total number of gray levels in the image, $n$ being the total number of pixels in the image, and $P_{x}(i)$ being in fact the image's histogram for pixel value $i$, normalized to [0,1] as presented by [37].

Unsharp Mask is used to sharpen the edges in the image. Without increasing noise or blemish the unsharp mask filter sharpens edges of the elements [38]. Digital unsharp masking is usually controlled by three settings:

Amount: the magnitude of each overshoot is controlled by amount and amount is recorded as a percentage.

Radius: the width of the edges is defined by radius.

Threshold: the minimum brightness change is handled by threshold that will be sharpened $[39,40]$.

Finally, thresholding is used to change the images to black and white figures to calculate the error. Thresholding changes each pixel in an image with a black pixel if the image intensity $I_{i j}$ is less than some fixed constant $\mathrm{T}$ or a white pixel if the image intensity is greater than that constant [41].

\section{EXPERIMENTAL WORK}

The vein locating systems have been designed to take images of the veins in hands/forearms using all infrared, near infrared and visual cameras. Technical specifications associated with infrared camera FLIR E25 are; resolution (thermal image quality) $=160 \times 120$, thermal sensitivity $<0.1{ }^{\circ} \mathrm{C}$, temperature range $=-20^{\circ} \mathrm{C}$ to $+350{ }^{\circ} \mathrm{C}$, built-in 2 Mpixels camera, spectral range $=7.5-13 \mu \mathrm{m}$, detector type $=$ Focal Plane Array (FPA), uncooled microbolometer $160 \times 129$ pixels, accuracy $=2 \%$ of temperature reading, built-in LED light, visual and thermal image fusion and image transfer to PC via USB. The thermal time constant is estimated to be about $12 \mathrm{~ms}$. An exact time constant value is not specified because there can be slight variations in the manufacturing process of the readout integrated circuits (ROICs) used in FLIR's uncooled devices. A 1.3 Mpixels Logitech QuickCam webcam has been used to capture visual images of $640 \times 480$ pxels. Field of view (FOV) is $60^{\circ}$, Focal Length $4.0 \mathrm{~mm}$, Optical Resolution (True) $1280 \mathrm{x}$ 960 1.2MP, Image Capture (16:9 W) 360p, 480p, 720p, Frame Rate (max) 30fps at 640x480. The near infrared CMOS-based sensor technology is used with plastic lens, wave length 700 to $900 \mathrm{~nm}$, field of view (FOV) is $60^{\circ}$, Focal Length $4.0 \mathrm{~mm}$, Optical Resolution (True) 1280 x 960 1.2MP, Image Capture (16:9 W) 360p, 480p, 720p, Frame Rate (max) 30fps at $640 \times 480$. The vein locators are connected to a laptop in which three software are used to capture data in the form of images which are AMCap for visual camera by Wintec, Quickcam for near infrared camera by Logitec and FLIR report 2.2 for high resolution (thermal) infrared camera FLIR E25. The above software are used only for the capturing of images from different types 
of cameras whereas, for the application of image processing techniques and finding errors (\%pixels) same software have been used for all images ( $\mathrm{N}=103)$; namely MATLAB, GIMP 2.8, Photoshop and DiffImg 2.2. Same image processing techniques have been applied on all 103 images of three different types.

The vein finding system consists of a camera holder/fixture with a rigid support to rest the subject's hand/arm, several types of cameras and an electronic circuit to control the intensities of visible-light LEDs and infrared LEDs for visual and near infrared images as shown in Figures 2 and 3 . The distance between the cameras and the rigid base upon which a subject places arm/hand is $28 \mathrm{~cm}$, which has been kept constant for capturing all images of three different types.

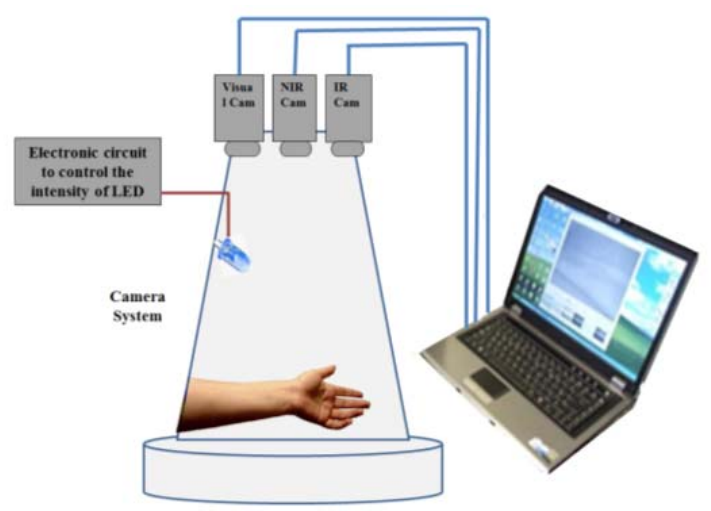

Fig. 2. Block diagram of the experimental set-up.

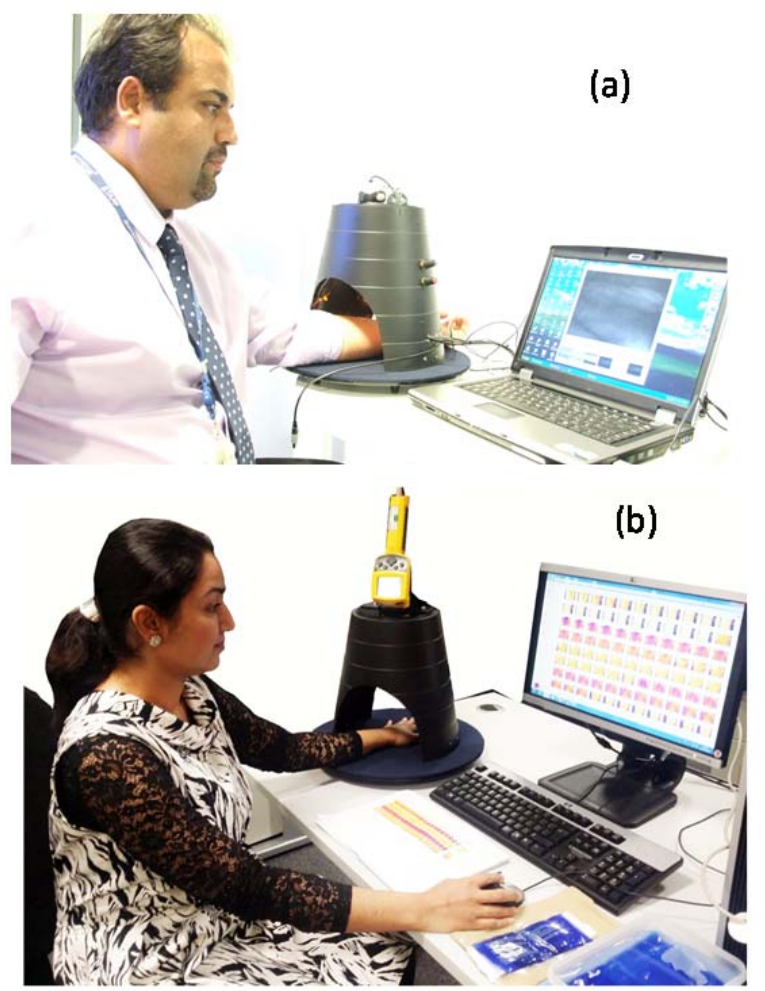

Fig. 3. A photo of the camera system with the laptop and the software; the near infrared and visual cameras (a), and FLIR E25 infrared camera (b).

\section{RESULTS}

Tables 2, 3 and 4 present some examples of the total images $(\mathrm{N}=103)$ of this study in the form of visual, infrared and near infrared images respectively. All images have been captured at the same distance from three different cameras $(28 \mathrm{~cm})$ with all possible combinations of intensities of visible-light and infrared LEDs especially for the visual and near infrared technologies. Total 103 images have been captured by three different types of cameras of volunteers associated with different characteristics such as, skin tone, ethnicities, gender, age and weight.

For visual and near infrared systems visible-light LEDs and infrared LEDs have been used for illumination purpose. A super pure yellow LED emits $7000 \mathrm{mcd}$ (millicandela) at $20 \mathrm{~mA}$ of current, which is the luminous intensity of the visible-light LED in this study. Whereas, the range of luminous intensity of infrared LEDs are between $16 \mathrm{~mW}$ (milliwatts) to $26 \mathrm{~mW}$ at 50mA. However, the terms 'high', 'low' and 'medium' in the Tables 2 and 4 refer to; an LED that gives off very (fully) bright light has a high luminous intensity, when an LED doesn't shine very brightly will have a lower luminous intensity and in between these two states will have a medium luminous intensity.

Infrared (thermal) images have been captured with and without the application of cold stimulation for all volunteers. There is no role of external LEDs in case of thermal images because FLIR E25 has built-in LED light.

\section{Table 2. Visual Images Data}

\begin{tabular}{|c|c|c|c|c|}
\hline $\begin{array}{c}\text { Intensity of } \\
\text { Visible-Light } \\
\text { LEDs }\end{array}$ & $\begin{array}{c}\text { Intensity of } \\
\text { Infrared } \\
\text { LEDs }\end{array}$ & \multicolumn{3}{|c|}{ Obtained Images } \\
\hline High & Low & & & \\
\hline High & High & & & \\
\hline Low & Low & & & \\
& & & & \\
\hline Low & High & & & \\
& & & & \\
& & & & \\
\hline
\end{tabular}


Table 3. Infrared Images Before and After Cold Stimulation

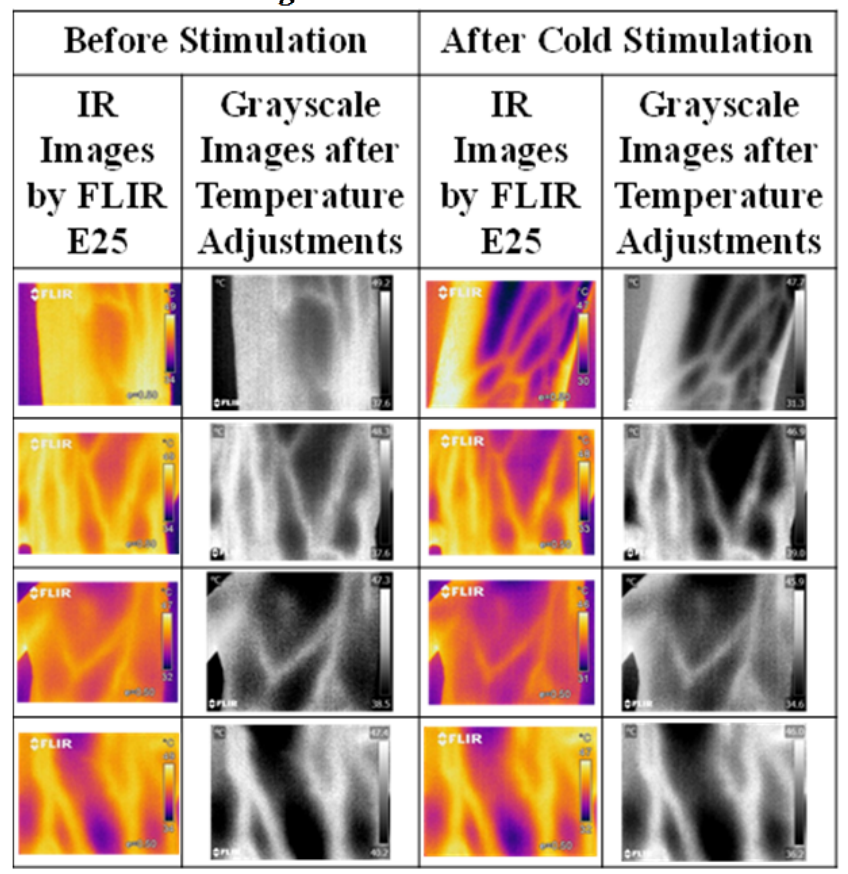

Table 4. Near Infrared Data for Different Intensity Combinations

\begin{tabular}{|c|c|c|c|c|}
\hline $\begin{array}{c}\text { Intensity of } \\
\text { Visible- } \\
\text { Light LEDs }\end{array}$ & $\begin{array}{c}\text { Intensity of } \\
\text { Infrared } \\
\text { LEDs }\end{array}$ & \multicolumn{3}{|c|}{ Captured Images } \\
\hline Low & Low & Low & & \\
\hline High & Low & & \\
\hline Medium & Low & & & \\
\hline Low & Medium & & & \\
\hline High & Medium & & & \\
\hline Medium & Medium & & & \\
\hline Low & High & & & \\
\hline Medium & High & & & \\
\hline
\end{tabular}

All captured images by three technologies including the sample images which are presented in Tables 2, 3 and 4 are processed by the previously suggested image processing techniques. An example of the application of the image processing techniques for near infrared data is illustrated in Figure 4.

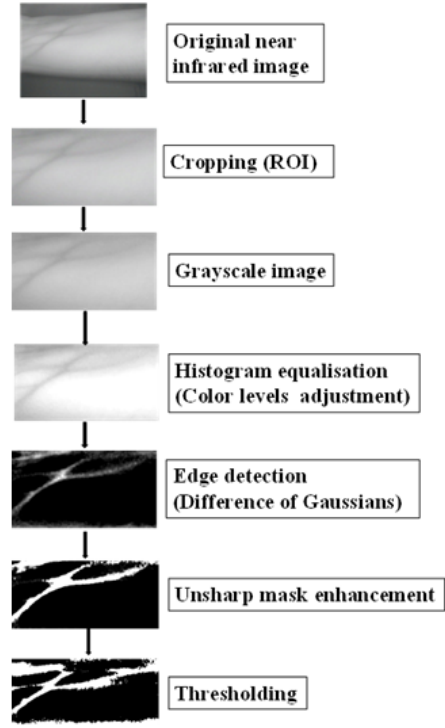

Fig. 4. Flow chart showing a sequence of image processing techniques applied on NIR image.

Firstly, the regions of interest are selected for all images. Secondly, all NIR and visual images are transformed into grayscale. Thirdly, colour levels are adjusted using histogram equalization technique because images are varied in contrast and brightness due to different combinations of LED's intensities. Fourthly, edge detection (difference of Gaussians) is applied after selecting smoothing parameters such as radius $1=250$ and radius $2=8.0$. Then, unsharp mask enhancement is performed on all NIR and visual images with the following chosen parameters such as radius $=380$, amount $=4.7$ and threshold $=38$. Finally, thresholding is performed on both types of images. The reason of selecting a set value for some of the parameters is to achieve the enhancement of the visualisation of the veins by image processing because best enhancement results has been found on these values.

For IR images, temperature range of thermal images is adjusted in FLIR report 2.2 to enhance the visualization of veins. Same techniques have been applied on IR images with slightly different parameters using the same soft-wares. After selecting ROI, infrared images are transformed into invert grayscale. Then, colour levels are adjusted using histogram equalization technique as in case of visual and near infrared images. Afterwards, edge detection (difference of Gaussians) is applied with the following smoothing parameters: radius $1=34$ and radius $2=17$. Here the values of the parameters are chosen differently due to the different nature of the thermal images but the main objective is the same which is to enhance the visualisation of the veins through image processing techniques. Similar to other images, unsharp mask enhancement is performed on IR images too. Finally, thresholding is performed.

Some of the examples of the processed NIR, visual and IR images after the application of image processing techniques are arranged in Table 5 for making a comparison between the three technologies to assess their suitability for the detection of veins. 
Table 5. Processed Near Infrared, Visual and Infrared Images for Comparison of Three Technologies

\begin{tabular}{|c|c|c|c|c|c|}
\hline \multicolumn{3}{|c|}{ Processed Near Infrared Images } & \multirow{3}{*}{$\begin{array}{c}\text { Pracessed } \\
\text { Visual Im ages }\end{array}$} & \multirow{2}{*}{\multicolumn{2}{|c|}{ Pracessed Infrared Images }} \\
\hline \multicolumn{2}{|c|}{$\begin{array}{c}\text { Intensity } \\
\text { Cambinations }\end{array}$} & \multirow[b]{2}{*}{$\begin{array}{l}\text { Processed Near } \\
\text { Infrared Im ages }\end{array}$} & & & \\
\hline \begin{tabular}{|c|} 
Visible \\
Light \\
LEDs \\
Intensity \\
\end{tabular} & $\begin{array}{c}\text { Infrared } \\
\text { LEDs } \\
\text { Intensity }\end{array}$ & & & $\begin{array}{c}\text { Before } \\
\text { Stimulation }\end{array}$ & $\begin{array}{l}\text { After Cold } \\
\text { Stimulation }\end{array}$ \\
\hline Low & Low & & & & \\
\hline High & Low & & & & \\
\hline Medium & Low & & & & \\
\hline Low & Medium & & & & \\
\hline High & Medium & & & & \\
\hline Medium & Medium & & & & \\
\hline Low & High & & & & \\
\hline High & High & & & & \\
\hline Medium & High & & & & \\
\hline
\end{tabular}

\section{A. Quantitative analysis of performance}

To assess the accuracy of three technologies to detect veins, a pattern of most probable visible veins (template) is generated manually for every single original image of all three types. Templates are converted into grayscale first and then thresholding is performed on them. Ultimately, a one to one comparison is made for every single image with its template image to find the differences. Consequently, the errors are calculated for testing the efficiency of the three technologies for the detection of veins. Some statistics such as error (\% pixels) for all images, mean error (\% pixels) for each technology and standard deviation of error (\% pixels) for each technology are calculated. Errors (\% pixels) for some of the visual, near infrared and infrared images are listed in Tables 6, 7 and 8 respectively along with the original images, templates and processed images.
Table 6. Visual Images with Error Calculations

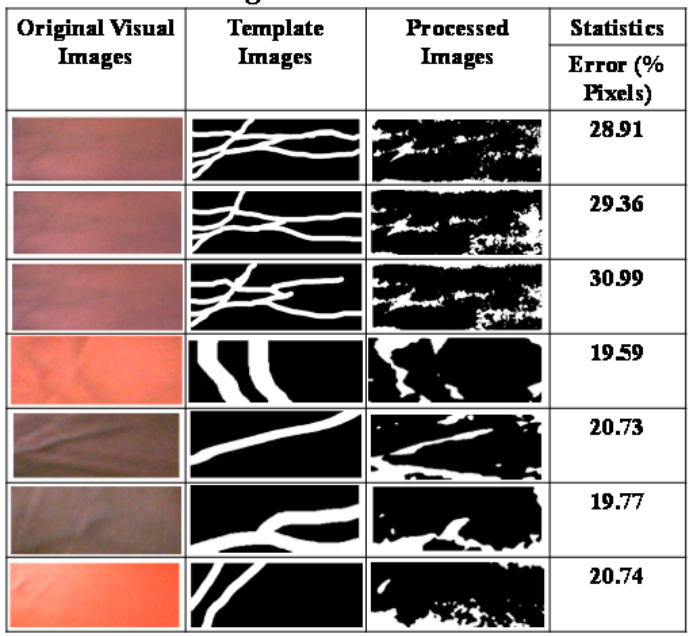

Table 7. Near Infrared Images with Error Calculations

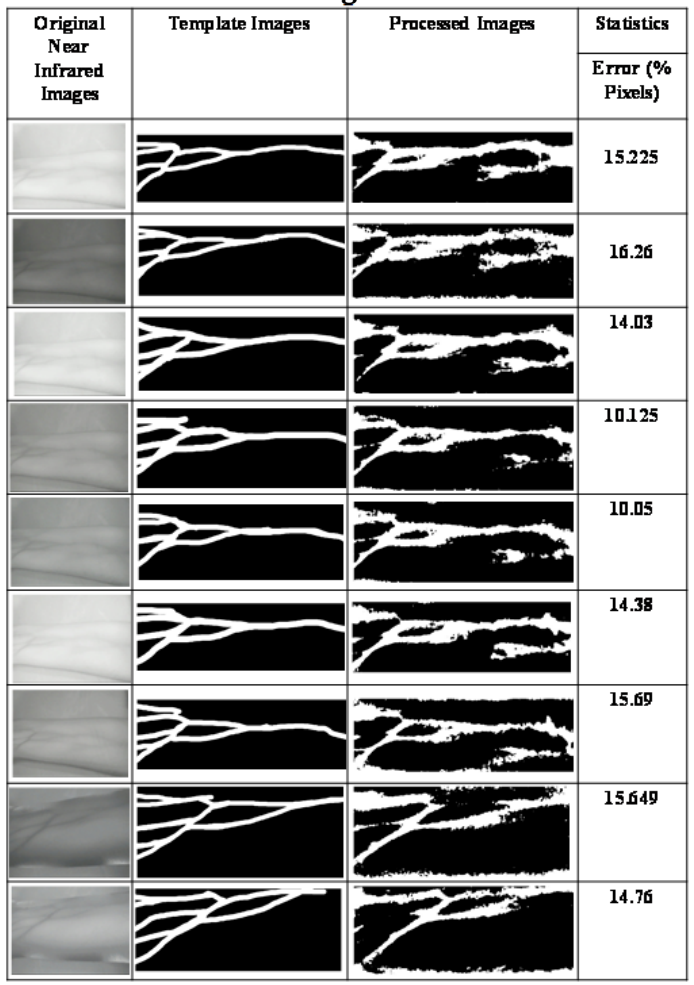


Table 8. Infrared Images Before and After Cold Stimulation with Error Calculations

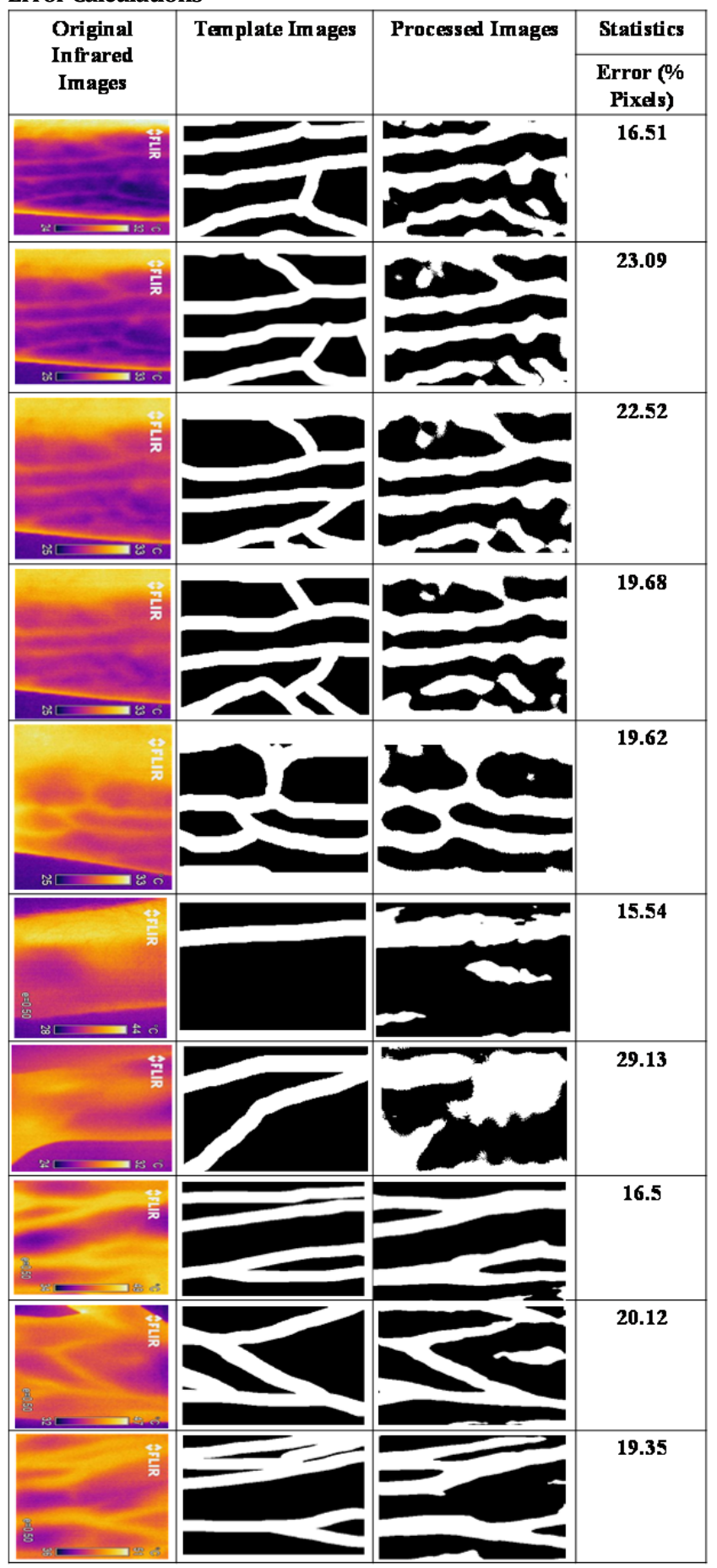

Thus, the errors (\% pixels) for all 103 images of all three types of the present study are calculated by comparing the processed image with its identification template in Difflmg. Mean errors (\% pixels) are calculated for each technology to assess their suitability for the detection of the veins. For visual images $(\mathrm{N}=38)$ mean errors $(\%$ pixels) are found to be 24.37 and standard deviation 3.55. For near infrared images $(\mathrm{N}=40)$ average errors (\% pixels) are calculated 17.16 and standard deviation 4.38. However, for infrared images $(\mathrm{N}=25)$ mean errors (\% pixels) are obtained 19.43 with standard deviation 4.51. The values of errors (\% pixels) for individual images of all three types can be seen in Figure 5. Figure 5 (a) indicates the values of error (\% pixels) for all visual images $(\mathrm{N}=38$ ), whereas error (\% pixels) for all near infrared images $(\mathrm{N}=40)$ can be seen in Figure 5 (b). Similarly, Figure 5 (c) shows the error (\% pixels) for all infrared images ( $\mathrm{N}=25)$. The tested number of images for three technologies are not identical because of different number of intensity combinations for visual and near infrared systems whereas thermal images are not intensity sensitive but influenced by temperature changes so those are captured with and without cold stimulation. So, the number of images for the three technologies vary but all three technologies cover the images of the same volunteers.

Hence, the experimental results are compared on the basis of statistics and plotted in the form of line graph and bar chart as shown in Figures 5 and 6. Mean error (\% pixels) for each technology and standard deviation of error (\% pixels) for each technology are calculated and displayed in Figure 6.

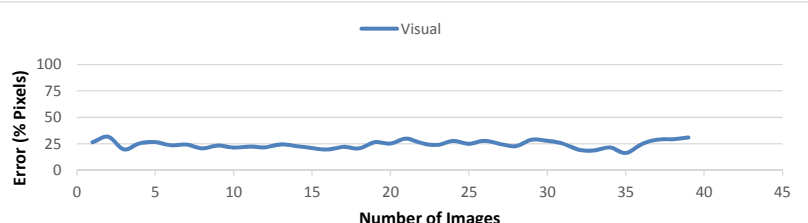

(a)

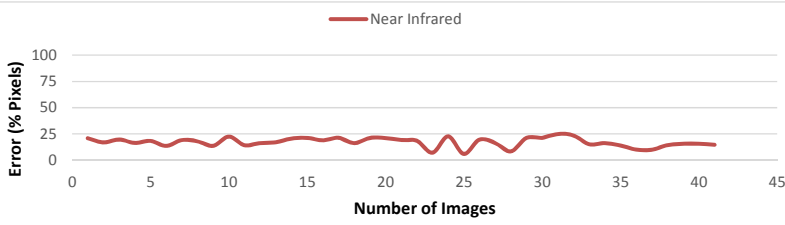

(b)

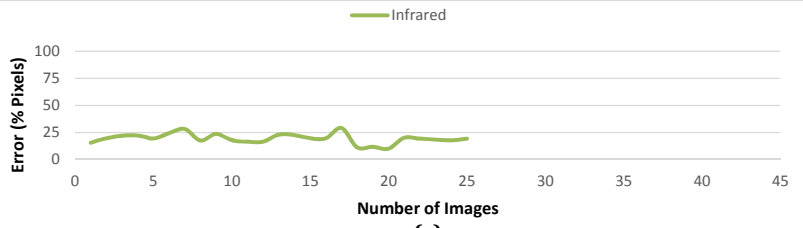

(c)

Fig. 5. Error level (\% pixels) for three types of technologies; visual (a), near infrared (b) and infrared systems (c).

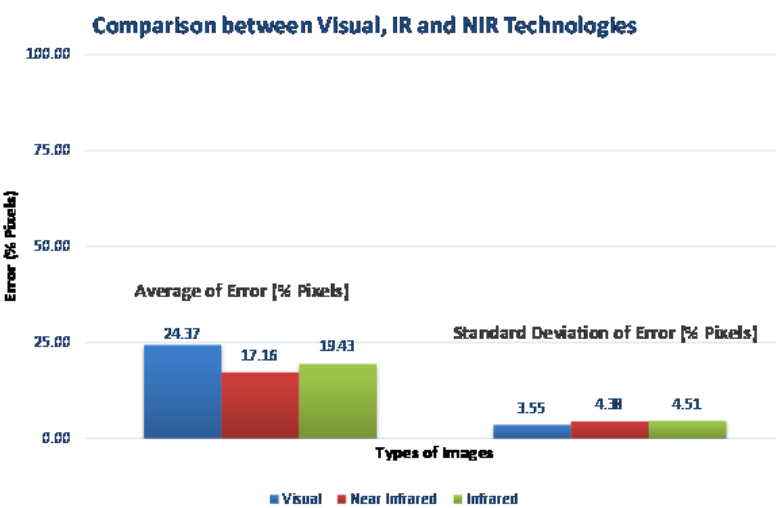

Fig. 6. Average error (\%pixels) of the three technologies and the associated standard deviation. 


\section{B. Discussion}

Qualitatively, it has been found that veins in NIR images of Table 4 can be seen clearly when compared to veins in visual and IR images of Tables 2 and 3 respectively. The most accurate results for the detection of veins are obtained by the near infrared camera at several combinations of intensities of visible-light and IR LEDs as shown in Tables 4 and 5. Similarly, quantitative analysis also suggests through statistical data that the calculated error (\% pixels) is found in case of NIR images to be the lowest in comparison with infrared and visual results.

The results for the visibility of veins for infrared images are obtained better when followed by cold compress. This gives an idea of introducing the hot stimulations in thermography in future to obtain better contrast in veins and the surrounding tissue for the visualisation purpose. Standard deviation of error (\% pixels) for infrared technology is found the highest because of the higher differences in error values (\% pixels) between the male and female subjects also before and after cold stimulation. Although, the average error (\% pixels) is the highest for visual images, the standard deviation of errors (\% pixels) is the lowest indicated high consistency of results.

\section{CONCLUSION}

This study has compared between visual, infrared and near infrared technologies for the detection of veins. It has been found that the visual cameras are less efficient for vein detection in comparison with the near infrared and infrared systems. However, the results of visual technology are found the most consistent but with higher error percentages.

Thus, it has been found qualitatively and quantitatively that the grayscale image processing combined with histogram equalisation, edge detection (difference of Gaussians), enhancement (unsharp mask) and thresholding make the near infrared technology supported by suitable visible-light LEDs and infrared LEDs intensities the most efficient technology to be used.

Hence, the results also show that the use of a cold compress (cold stimulation) for infrared technology helps to enhance the visualisation of veins.

\section{REFERENCES}

1. "The assessment Strategy for Cannulation and Venepuncture." [Online]. Available: http://www.ruh.nhs.uk/training/prospectus/clinical_skills/d ocuments/cannulation_and_venepuncture_workbook.doc. [Accessed: 25-Aug-2015].

2. S. J. Doniger, P. Ishimine, J. C. Fox, and J. T. Kanegaye, "Randomized Controlled Trial of Ultrasound-Guided Peripheral Intravenous Catheter Placement Versus Traditional Techniques in Difficult-Access Pediatric Patients," Pediatric Emergency Care, vol. 25, no. 3, pp. 154159, (2009).

3. http://www.terumo-europe.com/Relevant \%20Product\%20Info/Surflash_\%20First\%20Puncture\%20Suc cess\%20Study.pdf, [accessed on 25 August 2015].

4. T. G. Costantino, A. K. Parikh, W. A. Satz, and J. P. Fojtik, "Ultrasonography-Guided Peripheral Intravenous Access Versus Traditional Approaches in Patients With Difficult Intravenous Access," Annals of Emergency Medicine, vol. 46, no. 5, pp. 456-461, 2005.

5. J. M. Dargin, C. M. Rebholz, R. A. Lowenstein, P. M. Mitchell, and J. A. Feldman, "Ultrasonography-guided peripheral intravenous catheter survival in ED patients with difficult access," The American Journal of Emergency Medicine, vol. 28, no. 1, pp. 1-7, 2010.

6. J. J. Aponte, C. Menendez, D. Schellenberg, E. Kahigwa, H. Mshinda, P. Vountasou, M. Tanner, and P. L. Alonso, “Age Interactions in the Development of Naturally Acquired Immunity to Plasmodium falciparum and Its Clinical Presentation," PLoS Med PLoS Medicine, vol. 4, no. 7, 2007.

7. Mbamalu, David, and A. Banerjee. "Methods of obtaining peripheral venous access in difficult situations." Postgraduate medical journal 75.886: 459-462 (1999).

8. Mangold, Klaus, Joseph A. Shaw, and Michael Vollmer, "The physics of near-infrared photography", European Journal of Physics 34.6 (2013).

9. Stathopoulos, I., Skouroliakou, K., Michail, C., and Valais, I.Dynamic Infrared Thermography Study of Blood Flow Relative to Lower Limp Position. InJournal of Physics: Conference Series (Vol. 637, No. 1, p. 012027). IOP Publishing. 2015

10. Jung, Young-Jin, Jean Gonzalez, and Anuradha Godavarty. "Functional near-infrared imaging reconstruction based on spatiotemporal features: venous occlusion studies." Applied Optics 54.13 (2015): D82-D90.

11. E. Szufladowicz, R. Maniewski, E. Kozluk, A. Zbiec, A. Nosek, and F. Walczak, "Near-infrared spectroscopy in evaluation of cerebral oxygenation during vasovagal syncope," Physiol. Meas. Physiological Measurement, vol. 25, no. 4, pp. 823836, 2004.

12. Buono, M. J., Jechort, A., Marques, R., Smith, C., and Welch, J. "Comparison of infrared versus contact thermometry for measuring skin temperature during exercise in the heat." Physiological measurement, 28(8) (2007): 855.

13. . A. J. E. Bach, I. B. Stewart, G. M. Minett, and J. T. Costello, "Does the technique employed for skin temperature assessment alter outcomes? A systematic review," Physiol. Meas. Physiological Measurement, vol. 36, no. 9, Nov. 2015

14. V. C. Paquit, K. W. Tobin, J. R. Price, and F. Mèriaudeau, "3D and multispectral imaging for subcutaneous veins detection," Opt. Express Optics Express, vol. 17, no. 14, p. 11360, 2009.

15. J. Mangat, T. Standley, A. Prevost, J. Vasconcelos, and P. White, "A comparison of technologies used for estimation of body temperature," Physiol. Meas. Physiological Measurement, vol. 31, no. 9, pp. 1105-1118, 2010.

16. F Ring, A Jung and J Żuber Infrared Imaging. doi:10.1088/978-0-7503-1143-4 Published September 2015. Page 1-3 Copyright (C) IOP Publishing Ltd 2015. Online ISBN: 978-0-7503-1143-4 Print ISBN: 978-0-7503-1144-1 2015.

17. http://www.accuvein.com/products/catalog/av300-veinviewing-syste [accessed on 6 July 2014].

18. http://www.ebay.com/itm/AV300-Accuvein-Vein-Finder/181116630548 [accessed on 6 July 2014].

19. http://img.tjskl.org.cn/nimg/41/6a/8b7a318853ec0c2f678ef a44408b-98x98-

$1 /$ vein viewing medical light doctor costume accessories use safe light source no laser.jpg [accessed on 6 July 2014].

20. http://www.aliexpress.com/store/product/Upgraded-VeinViewing-System-Vein-Finder-Vein-LocatorBS2000/804433 625126805.html [accessed on 6 July 2014]. 
21. http://www.veinlite.com/purchasingagents/\#.U7vQqfldWmE [accessed on 6 July 2014].

22. http://www.veinlite.com/purchasingagents/\#.U7vQqfldWmE [accessed on 6 July 2014].

23. http://www.portable-ultrasound-scanner.com, [accessed on 27 August 2015]

24. http://www.tradesparq.com/products/1532687/Economical -Vein-Locator-manufacturers [accessed on 6 July 2014].

25. N. J. Cuper, J. H. Klaessens, J. E. Jaspers, R. D. Roode, H. J. Noordmans, J. C. D. Graaff, and R. M. Verdaasdonk, "The use of near-infrared light for safe and effective visualization of subsurface blood vessels to facilitate blood withdrawal in children," Medical Engineering \& Physics, vol. 35, no. 4, pp. 433-440, 2013.

26. http://www.yueshenyl.cn/product/1896497141$\underline{221137422 / \text { hot sale portable vascu luminator device.ht }}$ ml. [Accessed on 5 July 2014].

27. http://www.ohgizmo.com/2006/11/15/luminetxveinviewer/\#!baJYx2 [Accessed on 5 July 2014].

28. http://www.techeblog.com/index.php/tech-gadget/25000vein-viewer-by-luminetx [Accessed on 5 July 2014].

29. http://www.vision-systems.com [Accessed on 5 July 2014].

30. http://www.vuetekscientific.com/features-benefits.html [accessed on 13 November 2015].

31. http://www.itv.com/news/central/2015-10-13/the-mobilephone-which-could-reduce-botched-injections/[Accessed on 13 November 2015].

32. V. C. Paquit, F. Meriaudeau, J. R. Price, and K.W. Tobin, "Simulation of skin reflectance images using 3D tissue modeling and multispectral Monte Carlo light propagation," in 30th Annual International Conference of the IEEE Engineering in Medicine and Biology Society, 2008. EMBS 2008, pp. 447-450 (2008).

33. E. F. J. Ring and K. Ammer, "Infrared thermal imaging in medicine," Physiol. Meas. Physiological Measurement, vol. 33, no. 3, 2012.

34. Zhang, X., Liu, X. S., Yang, X. R., Chen, S. L., Zhu, P. P., \& Yuan, Q. X.. Mouse blood vessel imaging by in-line $x$-ray phase-contrast imaging.Physics in medicine and biology, 53(20), 5735 (2008)

35. http://www.johndcook.com/blog/2009/08/24/algorithmsconvert-color-grayscale/ Cook, J. "Three algorithms for converting color to grayscale." The Endeavour (2009).

36. Lindeberg, T. Image matching using generalized scale-space interest points (pp. 355-367). Springer Berlin Heidelberg (2013).

37. Russ, J. C. The image processing handbook. CRC press (2011).

38. http://www.cambridgeincolour.com/tutorials/imagesharpening.htm [Accessed on 13 November 2015].

39. http://www.scantips.com/simple6.html[Accessed on 13 November 2015].

40. K. N. Jabri and D. L. Wilson, "Quantitative assessment of image quality enhancement due to unsharp-mask processing in x-ray fluoroscopy," Journal of the Optical Society of America A J. Opt. Soc. Am. A, vol. 19, no. 7, p. 1297, Jan. 2002.

41. Y.-K. Lai and P. L. Rosin, "Efficient Circular Thresholding," IEEE Transactions on Image Processing IEEE Trans. on Image Process., vol. 23, no. 3, pp. 992-1001, 2014. 